\title{
ANÁLISIS LÍTICO DE OCUPACIONES DEL HOLOCENO MEDIO DE HORNILLOS 2 (JUJUY, ARGENTINA): DISCUTIENDO LA TECNOLOGÍA Y DISTRIBUCIÓN DE LAS PUNTAS DE PROYECTIL "SAN MARTÍN"
}

\author{
LITHIC ANALYSIS OF MIDDLE HOLOCENE OCCUPATIONS FROM \\ HORNILLOS 2 (JUJUY, ARGENTINA): DISCUSSING THE TECHNOLOGY \\ AND DISTRIBUTION OF "SAN MARTÍN" PROJECTILE POINTS
}

\author{
Rodolphe Hoguin ${ }^{1}$ y Hugo D. Yacobaccio ${ }^{2}$
}

\begin{abstract}
En este artículo se analiza el material lítico de la capa 3 del alero Hornillos 2 (Susques, Pcia. de Jujuy, Argentina) fechada en los inicios del Holoceno Medio (7760-7430 a.p.), asociada con puntas "San Martín". Este tipo de punta, rara vez mencionada en la arqueología de la vertiente oriental de la Puna de Atacama, tiene una importante distribución en el Salar de Atacama, cuenca superior del río Loa y en otras márgenes de salares en el norte de Chile. El análisis del material lítico muestra que las ocupaciones de los comienzos del Holoceno Medio en el sitio son de corto plazo y de actividades especializadas en el marco de una organización tecnológica relacionada con una movilidad logística. En este contexto, las puntas San Martín han tenido una larga vida útil y múltiples secuencias de manufactura.
\end{abstract}

Palabras claves: Holoceno Medio, tecnología lítica, puntas “San Martín”, Puna de Atacama.

The goal of this paper is to analyze the lithic artefacts from layer 3 of Hornillos 2, a rockshelter located at Susques (Jujuy, Argentina), which have been dated to the beginning of the Middle Holocene (7,760-7,430 BP). These include points of the "San Martín" type, rarely mentioned in Argentine archaeology, but with a broad distribution around the Salar de Atacama and other salt-lake margins in Northern Chile, as well as the upper drainage of the Loa River. Lithic analysis shows that the early Middle Holocene occupations of the site are short term, reflecting specialized activities and a technological organization related to logistical mobility. In this context, San Martín points had a long history involving multiple manufacture sequences.

Key words: Middle Holocene, lithic technology, San Martín points, Puna de Atacama.

Este trabajo presenta los resultados del análisis lítico de la capa 3 del alero Hornillos 2 (Susques, Pcia. de Jujuy, Figura 1) de la cual se recuperaron puntas tetragonales identificadas recientemente en la bibliografía chilena como tipo "San Martín" (Núñez et al. 2005), antes denominadas "Tambillo" (Le Paige 1970; Schobinger 1969). Esta ocupación ha sido datada en Hornillos 2 en 7.430 \pm 80 a.p. (UGA-7830), carbón, y 7.760 160 a.p. (UGA8722), carbón (Yacobaccio et al. 2005).

La particularidad de este caso de estudio es que el tipo morfológico de cabezal lítico asociado a estos contextos presenta una alta dispersión en varios lugares de Chile (Le Paige 1970; Núñez et al. 2005) y de la Puna argentina (Schobinger 1969; Schobinger et al. 1974; Yacobaccio et al. 2005), aunque nunca se analizaron las razones de su ausencia en sitios de la Puna oriental de Jujuy, ni al sur del límite entre la Puna Seca y la Puna Salada. Más allá de las similitudes generales entre el material chileno y argentino, este tipo morfológico de punta de proyectil podría presentar variabilidad desde el punto de vista técnico. Entonces, los objetivos centrales de esta contribución se orientan a caracterizar el material lítico asociado con estos cabezales presentes en la capa 3 del sitio mencionado (Hornillos 2); a reconstruir las distintas secuencias de talla realizadas e interpretar el rol del sitio en el marco de una organización tecnológica llevada a cabo en la región, infiriendo cadenas operativas (sensu Inizan et al. 1995), y a comparar los resultados obtenidos con los de otros sitios de la región y del norte de Chile.

\footnotetext{
1 Instituto de Arqueología, Universidad de Buenos Aires y CONICET, Buenos Aires, Argentina. Maison de l'Archéologie et de l'Ethnologie, Université Paris X y CNRS UMR7041, Nanterre, France. roddh2002@ yahoo.fr

2 Instituto de Arqueología, Universidad de Buenos Aires y CONICET, Buenos Aires, Argentina. hdyacobaccio@gmail.com
} 


\section{Antecedentes}

Los inicios del Holoceno Medio están marcados por la instalación de condiciones áridas y por un probable aumento de temperatura que llegó a su máximo alrededor de 6.000 a.p. (Núñez y Grosjean 1994). Sin embargo, localmente, en el área de Susques, las condiciones húmedas del Holoceno Temprano continuaron, al menos hasta los 7.000 a.p., aunque registrando fluctuaciones tales como un pulso seco en torno al 8.300 a.p. (Morales 2010; Yacobaccio y Morales 2005). En Chile se registró también una disminución notable del nivel de varios lagos entre el 8.400 y el 8.000 a.p. involucrando una baja en la oferta de recursos (Núñez et al. 1997). En distintos sitios se pudieron observar hiatos en las secuencias de ocupación o una disminución de la intensidad en las mismas (Núñez et al. 2002). Debido a la segmentación del ambiente y del contraste entre las áreas de alta y baja productividad se propuso que la movilidad residencial decreció y que las poblaciones permanecieron en torno a los parches más productivos (Aschero 1994; Morales 2010; Núñez y Grosjean 1994; Yacobaccio y Morales 2005). De esta manera concomitante, parecen haberse desarrollado estrategias de movilidad logísticas por parte de los cazadores recolectores que poblaban la región (Pintar 1995). En la Puna Salada de Argentina se propuso el aglutinamiento estacional o permanente para realizar cazas colectivas. Estas cazas fueron realizadas mediante nuevas técnicas utilizadas simultáneamente, resultando en una diversificación de morfologías de las puntas de proyectil (Aschero y Martínez 2001).

Como se ha mencionado líneas arriba, en los inicios del Holoceno Medio se ha registrado la presencia de puntas tetragonales (Figura 2) en varios lugares de la Puna Argentina, como Hornillos 2, terrazas de Quebrada Lapao y El Aguilar (Hoguin 2010; Schobinger et al. 1974; Yacobaccio et al. 2005). De la misma manera, fueron registradas, aunque en este caso datadas en torno a los 8.210 a.p. y 8.130 a.p., en el salar de Tuyajto al este del salar de Atacama, así como en el norte del Loa en sitios tales como San Martín 4, Ollargüe/E, Minchincha-1 y Ascotán 4A, entre otros (Núñez et al. 2005). Las puntas tetragonales no son comunes en el sitio Tambillo 1 , aunque son más frecuentes en sitios aledaños (Ta-3A, B, 4 y 5) (Núñez et al. 2005: 80). En el sitio San Martín 4A fueron datadas en 8.070 \pm 50 a.p. En ambas vertientes este tipo de punta se asocia a otras morfologías, principalmente triangulares y foliáceas. Cabe destacar que los fechados obtenidos en Hornillos 2 son inmediatamente posteriores al inicio del Holoceno Medio, lo que es llamativo en términos de continuidad espacial y temporal. Así, tal dispersión geográfica y estas diferencias cronológicas merecen ser analizadas para tratar de aclarar cuáles fueron los eventos responsables de tal patrón de distribución de estas puntas "San Martín". En cuanto al aspecto tecnológico, en otro trabajo ya se ha resaltado cierta estandarización morfológica y métrica de estas puntas y la variabilidad presente en la manera de retocarlas (Hoguin 2010). Allí se ha planteado que esta variación en las modalidades técnicas estaría relacionada con la implementación del saber-hacer y de las habilidades individuales de los talladores (sensu Pelegrin 1995).

\section{Aspectos Teóricos}

Con el objetivo de reconstruir las cadenas operativas relacionadas a los artefactos hallados en la capa 3 del alero Hornillos 2, se intentará un acercamiento al sistema económico así como a los comportamientos técnicos relacionados con la talla. El primer paso de este tipo de investigación se centra sobre las estrategias de aprovisionamiento de materias primas, se tienen en cuenta el origen geográfico de las rocas, su disponibilidad y las estrategias de abastecimiento -indagando bajo qué forma circularon las materias primas- (Inizan et al. 1995) para considerar distintas cuestiones. En primera instancia, la simple ausencia o presencia de una materia prima es informativa sobre los comportamientos en cuanto a la organización tecnológica, particularmente considerando rangos de aprovisionamiento a escala de sitio (Gamble 1992). Asimismo, es importante tomar en cuenta los distintos aspectos geomorfológicos acerca de las fuentes de materias primas como la abundancia y dispersión de las rocas, que resulta ser uno de los factores que restringen las elecciones de tipo económico. En este sentido, la abundancia se refiere a la cantidad de materia prima aprovechable en una fuente, mientras que la dispersión se refiere a cuán esparcidos están los bloques o nódulos en el paisaje. Ciertas restricciones están impuestas por la forma bajo la cual se presentan los bloques, por eso, siempre se deben comparar estos aspectos de las fuentes con los materiales hallados en los sitios (Inizan et al. 1995). 


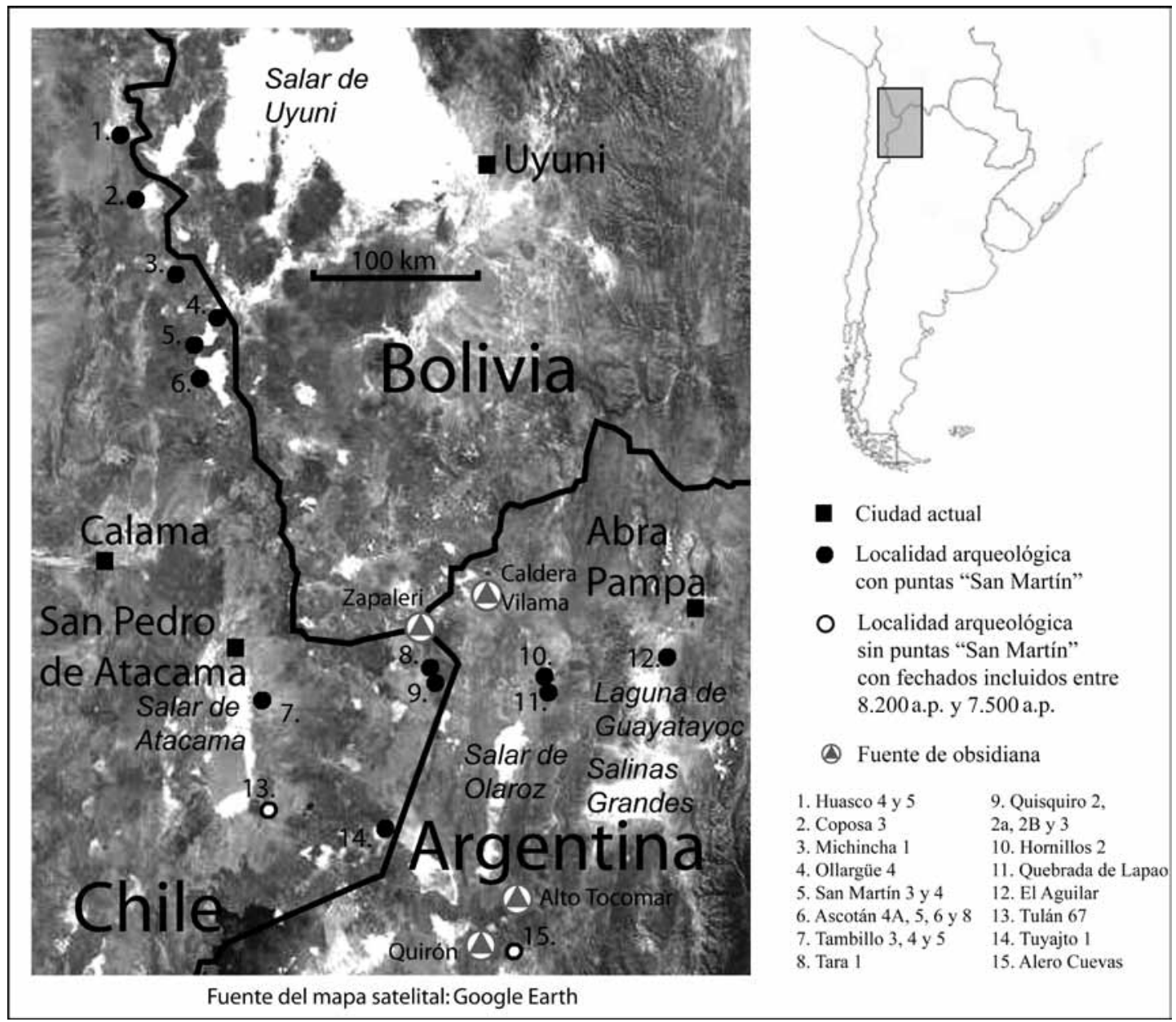

Figura 1. Localización de los sitios con puntas "San Martín" o con cronología contemporánea en el Norte de Chile y Noroeste Argentino.

Location of sites with "San Martín" points, or sites with contemporary occupations, in Northern Chile and NW Argentina.

De esta forma, se trata entonces de pensar cómo se organizó el aprovisionamiento, cuáles fueron las tareas destinadas a la producción de instrumentos y dónde se realizaron, dado que las distintas etapas de reducción pueden, o no, desarrollarse en distintas localidades siguiendo decisiones tecnológicas y económicas (Inizan et al. 1995). La organización de la tecnología forma parte fundamental de las estrategias de movilidad, particularmente en función de anticipar las necesidades de una localidad donde los individuos piensan quedarse más o menos tiempo (Kent 1992). En el caso de la tecnología lítica, el transporte de un equipo instrumental se realizaría si el lugar está desprovisto de las rocas requeridas por algunas tecnologías, o si el tiempo de estadía es corto. En estos casos, el equipo instrumental suele haber sido confeccionado a partir de materias primas que no están disponibles en la localidad. Además, si el tiempo de asentamiento es corto, es esperable que la proporción de instrumentos sea importante en relación a los desechos (Kent 1992). Los datos etnográficos (Binford 1980; Kent 1992) permiten entender algunos factores que intervienen en la formación del registro arqueológico y particularmente en la del material lítico hallado: por ejemplo, es necesario determinar los artefactos que fueron confeccionados in situ, cuya existencia puede estar solamente reflejada por los desechos. Esto permite comprobar si hubo traslado y abandono de artefactos en el mismo sitio que no están exclusivamente relacionados con los materiales presentes, salvo si se considerara una organización tecnológica en la cual se asume 

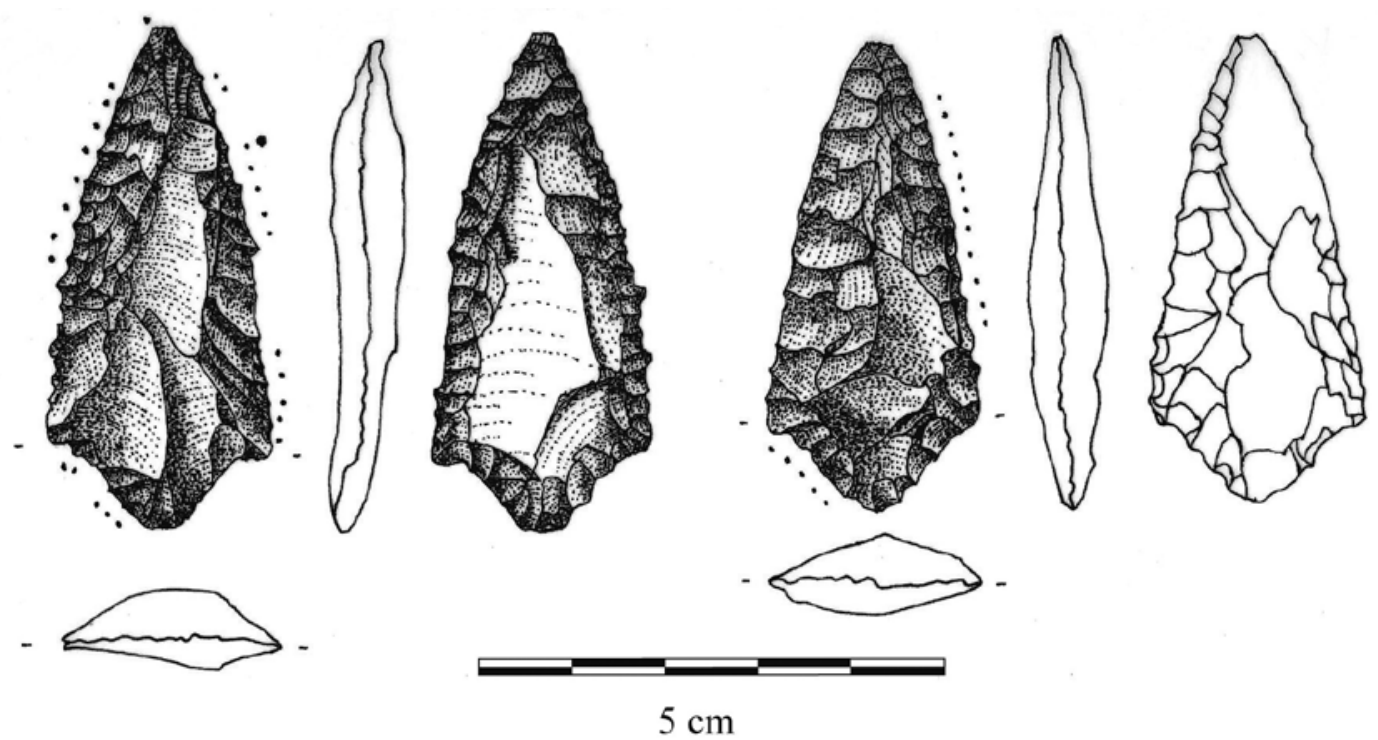

Figura 2. Puntas de proyectil tetragonales "San Martín" de Quebrada de Lapao.

Tetragonal "San Martín" projectile points from Quebrada de Lapao.

que la ocupación del sitio estuvo relacionada con un aprovisionamiento y descarte en un rango de acción principalmente local.

Un estudio más fino sobre los comportamientos técnicos requiere también evidenciar un proyecto de talla, a través de esquemas operativos realizados mediante métodos y técnicas. La técnica se refiere al tipo de percusión y el gesto motor que lo acompaña, y el método se entiende como el encadenamiento de dichos gestos (Inizan et al. 1995; Pelegrin 1995). Para reconstruir estas etapas es necesario pasar por diversas instancias de experimentación y remontaje entre distintos artefactos. Sin embargo, en su defecto, los remontajes mentales a partir de métodos de representaciones gráficas y de análisis estadísticos ofrecen la posibilidad de reconstruir este proyecto de talla.

El aspecto cognitivo de las cadenas operativas, los esquemas operativos y conceptuales, son muy útiles en términos de entender las conductas de los artesanos (Boëda 1997). En efecto, el proyecto de talla involucra actos predeterminados. Su realización requiere de habilidades motrices particulares, del desarrollo de un saber-hacer (adquirido por la práctica), y de la adquisición de conocimientos de orden intelectual (Boëda 1997; Inizan et al. 1995; Pelegrin 1995). Los estudios cognitivos permitieron evidenciar elecciones y restricciones de distinta naturaleza cultural y económica, con distintos grados de habilidades motrices y mentales (Inizan et al. 1995; Pelegrin 1995) o con una flexibilidad más o menos restringida hacia hábitos técnicos de los grupos, pudiendo reflejar comportamientos más o menos estables a través del tiempo (Boëda 1997; Hoguin 2010; Pelegrin 1995). La reflexión sobre estos distintos planteos, y de cómo están relacionados con diversos aspectos organizativos como la movilidad, el tamaño de los grupos y la territorialidad, permite entonces pensar la planificación tecnológica a distintos niveles de análisis.

\section{Consideraciones Metodológicas y Terminológicas}

Una vez que el conjunto lítico está separado por materias primas, una clasificación en grandes tipos de productos permitirá reflejar en una primera instancia la organización tecnológica en términos generales. Lo primero que se observa al respecto es el nivel de descortezamiento, que se refiere a las primeras lascas talladas a partir de un núcleo. En una segunda instancia se estudiarán más detalladamente las otras lascas que presentan distinto grado de reserva de corteza. Se hablará de lascas predeterminando o predeterminadas (Boëda 1997) cuando se trate de aquellas lascas que fueron obtenidas con el objetivo de conseguir formas bases (predeterminando) o para referirse a las 
formas-bases en sí mismas (predeterminadas). Las lascas de adelgazamiento se definen como aquellas destinadas a formatizar las piezas en relación a su sección, mientras que las de retoque son aquellas que formatizaron las piezas según un eje bilateral. Fueron clasificadas como lascas de reactivación aquellas que presentan rastros complementarios en la sección talón-cara dorsal, así como las que presentan muchas extracciones regulares (igual o mayor a 5), centrípetas y/o escalonadas.

En un segundo paso, se toman en cuenta tres aspectos para interpretar la distribución de materias primas: 1) el número de piezas $(\mathrm{N}), 2$ ) el número mínimo de desechos (NMD) considerando la cantidad de partes proximales y las piezas enteras y 3) el peso (g). Es importante considerar este último dentro de un enfoque tecno-económico porque la alta cantidad de desechos no refleja especialmente una actividad dispendiosa para una materia prima dada si el peso de los desechos es muy bajo.

Luego el remontaje mental (Pelegrin 1995) requiere considerar las dimensiones de los productos, las extracciones y los tipos de talones. Resumiremos las dimensiones de la siguiente forma: un tamaño promedio longitud/ancho [(largo+ancho)/2] y los módulos (largo/ancho). Las frecuencias de estos dos últimos serán presentadas bajo forma de histogramas de distribución de barras realizados con el programa estadístico PAST. Debemos aclarar que no presentaremos los tipos de extracciones de arista simple, ni las transversales o angulares (sensu Aschero 1983), que no son diagnósticas de un método de talla en particular. Por el contrario, las extracciones paralelas sí pueden reflejar métodos laminares y/o técnicas de adelgazamiento por presión, y las centrípetas, discoidales y/o de adelgazamiento bifacial. Los talones diedros y facetados serán agrupados juntos y luego tomados como posibles indicadores de formatización bifacial (Aschero 1983). Se considerarán también los talones puntiformes, relacionados con la técnica de presión, y los lisos mayores a $4 \mathrm{~mm}$ con estrías, conos y otros indicadores de percusión directa dura (Marchand 1999).

\section{Las Materias Primas}

Las principales características geomorfológicas de las fuentes de materias primas que se describen a continuación están resumidas en la Tabla 1. En Susques afloran importantes filones de cuarcita en las Sierras del Taire (fuentes primarias). Allí, los bloques son de todos los tamaños y con aristas muy salientes, ofreciendo plataformas y superficies de talla inicialmente cómodas. Se ubican regularmente en el paisaje, en varias localizaciones discontinuas. Los efectos de pendiente y ríos dispersaron estos bloques a través de toda el área (fuentes secundarias). En este caso, los rodados tienden a ser más pequeños y patinados.

En cuanto a las obsidianas la determinación geoquímica fue objeto de estudios en los últimos años. Particularmente, la determinación de fuentes de obsidiana en el Noroeste permitió ver patrones de circulación de esta roca (Yacobaccio 2010; Yacobaccio et al. 2004). Para la región de estudio, nos interesan particularmente las localidades siguientes por presentar variantes geoquímicas conspicuas: Zapaleri/Laguna Blanca, Caldera Vilama, Alto Tocomar, Quirón y Ona. Estos vidrios volcánicos se presentan bajo forma de nódulos pequeños, aunque pudiendo alcanzar $20 \mathrm{~cm}$ en algunas fuentes como la de Zapaleri (Yacobaccio et al. 2004). Estas fuentes primarias están concentradas en el paisaje y no aparecen dispersas en fuentes secundarias, salvo en Alto Tocomar por lo que sabemos hasta ahora.

En cuanto a la andesita, se trata de una roca volcánica para la cual solo se conoce una fuente principal a $\sim 40 \mathrm{~km}$ al Oeste de Hornillos 2, constituida por una extensa cantera-taller en la localidad

Tabla 1. Propiedades de las fuentes de materia prima.

Raw material source properties.

\begin{tabular}{lcccccc}
\hline Materia Prima & $\begin{array}{c}\text { Abundancia de } \\
\text { bloques }\end{array}$ & Dispersión & $\begin{array}{c}\text { Tamaño de los } \\
\text { bloques }\end{array}$ & $\begin{array}{c}\text { Presentación de } \\
\text { las rocas }\end{array}$ & Distancia & Calidad \\
\hline Cuarcita fuentes primarias & Muy alta & Alta & Hasta $50 \mathrm{~cm}$ & Lajas & $1 \mathrm{~km}$ & Mediana \\
Cuarcita fuentes secundarias & Mediana & Muy alta & Hasta $20 \mathrm{~cm}$ & Nodulos $/$ rodados & $0 \mathrm{~km}$ & $\begin{array}{c}\text { Mediana } \\
\text { Buna }\end{array}$ \\
Andesita & Muy alta & Baja & Hasta $100 \mathrm{~cm}$ & Bloques & $50 \mathrm{~km}$ & $\begin{array}{c}\text { Buena } \\
\text { Obsidiana }\end{array}$ \\
Muy alta & Baja & Hasta $20 \mathrm{~cm}$ & Nodulos/rodados & $100 \mathrm{~km}$ & Muy buena \\
Otras & Baja & Muy baja & - & Variable & $10 \mathrm{~km} ?$ & Variable \\
\hline
\end{tabular}


de El Toro. Allí se presenta en una alta diversidad de bloques de distintos tamaños, algunos muy grandes, otros con extracciones y una concentración de artefactos asociada a los mismos, arrastrados por los ríos Toro y Rosario. Una posible fuente primaria de estos bloques estaría ubicada en las cercanías del cerro Zapaleri.

Varios filones de otras rocas más restringidos y menos frecuentes se pueden encontrar localmente. Se trata de varios tipos de rocas silíceas: riolita, cuarcita de grano fino, calcedonia, ftanita, ópalo, entre otros.

\section{Las Actividades de Talla}

A primera vista, el hecho sobresaliente de la Tabla 2, es la muy baja cantidad de lascas de descortezamiento. Los núcleos hallados en esta capa son de pequeñas dimensiones y uno de ellos fue reciclado. Este último es de riolita (roca silícea) y no fue tomado en cuenta en el total de la muestra porque fue también contabilizado como instrumento (que fue su función última antes del descarte). La cantidad de instrumentos y de lascas predeterminadas es bastante baja. Lo que claramente predomina son las lascas de adelgazamiento y de retoque, y luego las lascas indiferenciadas y aquellas de reactivación para algunas de las materias primas. Resultan notables también los relativamente altos porcentajes de lascas de reactivación para rocas como la andesita (18\%), la obsidiana (15\%) y la cuarcita de grano fino $(21 \%)$, y de adelgazamiento para la obsidiana (26\%), las rocas silíceas $(22 \%)$ y la cuarcita de grano fino $(21 \%)$.

El número total de desechos es bastante representativo de las proporciones del número mínimo de

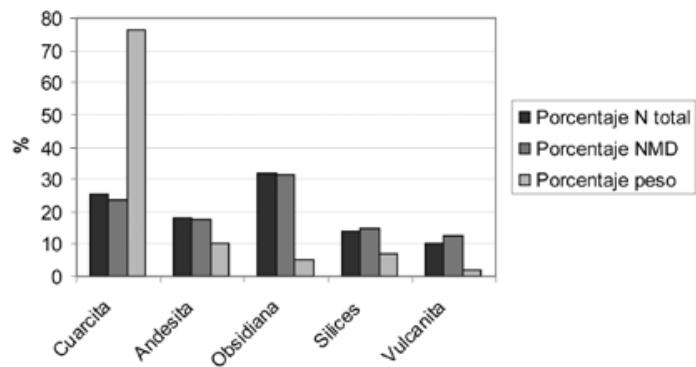

Figura 3. Porcentaje de cada materia prima por N, NMD y peso. Proportion of each raw material by N, NMD and weight.

desechos (Figura 3), lo cual podría ser el resultado de una buena preservación tafonómica de las piezas. Si en proporción la obsidiana domina el conjunto por su N y por su NMD, sin embargo la cuarcita está mejor representada en el sitio si nos basamos en el peso.

En cuanto a los tamaños de los artefactos, cabe notar que las mayores frecuencias corresponden a artefactos menores a $20 \mathrm{~mm}$ para la cuarcita (Figura 4.1), menores a $12 \mathrm{~mm}$ para la andesita (Figura 4.2) y menores a $10 \mathrm{~mm}$ para las otras materias primas (Figuras 4.3, 4.4 y 4.5). Se puede, sin embargo, mencionar la presencia destacable de una amplia variedad de tamaños para la cuarcita (entre 32 y $72 \mathrm{~mm}$ ), aunque aún en muy baja frecuencia. En cuanto a los módulos obtenidos, se puede observar que la mayor parte de ellos es inferior a 1,5 para la cuarcita con una importante proporción inferior a 1 (Figura 5.1). Se puede destacar, también, la baja frecuencia de módulos alargados o laminares (superior o igual a 2). Las mismas tendencias se pueden observar para la andesita pero con una ausencia total de módulos laminares (Figura 5.2). Respecto de la

Tabla 2. Muestra analizada clasificada por productos y por materia prima. Sample analyzed and classified by products and raw material.

\begin{tabular}{|c|c|c|c|c|c|c|c|c|c|c|c|}
\hline \multirow{2}{*}{ Tipo de artefato } & \multicolumn{2}{|c|}{ Cuarcita } & \multicolumn{2}{|c|}{ Andesita } & \multicolumn{2}{|c|}{ Obsidiana } & \multicolumn{2}{|c|}{ Rocas silíceas } & \multicolumn{2}{|c|}{ Cuarcita de grano fino } & \multirow{2}{*}{$\frac{\text { Total }}{\mathrm{N}}$} \\
\hline & $\mathrm{N}$ & $\%$ & $\mathrm{~N}$ & $\%$ & $\mathrm{~N}$ & $\%$ & $\mathrm{~N}$ & $\%$ & $\mathrm{~N}$ & $\%$ & \\
\hline Descortezamiento & 2 & 1 & & & & & & & & & 2 \\
\hline Predetermin. & 10 & 5 & 2 & 2 & 4 & 2 & 1 & 1 & 2 & 3 & 19 \\
\hline Adelgazamiento & 19 & 10 & 15 & 12 & 63 & 26 & 23 & 22 & 16 & 21 & 136 \\
\hline Reactivación & 18 & 10 & 23 & 18 & 36 & 15 & 10 & 9 & 16 & 21 & 103 \\
\hline Retoque & 79 & 42 & 60 & 48 & 104 & 44 & 55 & 52 & 34 & 45 & 332 \\
\hline Indiferenciado & 54 & 29 & 20 & 16 & 27 & 11 & 14 & 13 & 7 & 9 & 122 \\
\hline Instrumentos & 5 & 3 & 4 & 3 & 2 & 1 & 3 & 3 & 1 & 1 & 15 \\
\hline Núcleos & 1 & 1 & & & & & 1 & 1 & & & 2 \\
\hline Total & 188 & & 126 & & 239 & & 106 & & 75 & & 729 \\
\hline
\end{tabular}



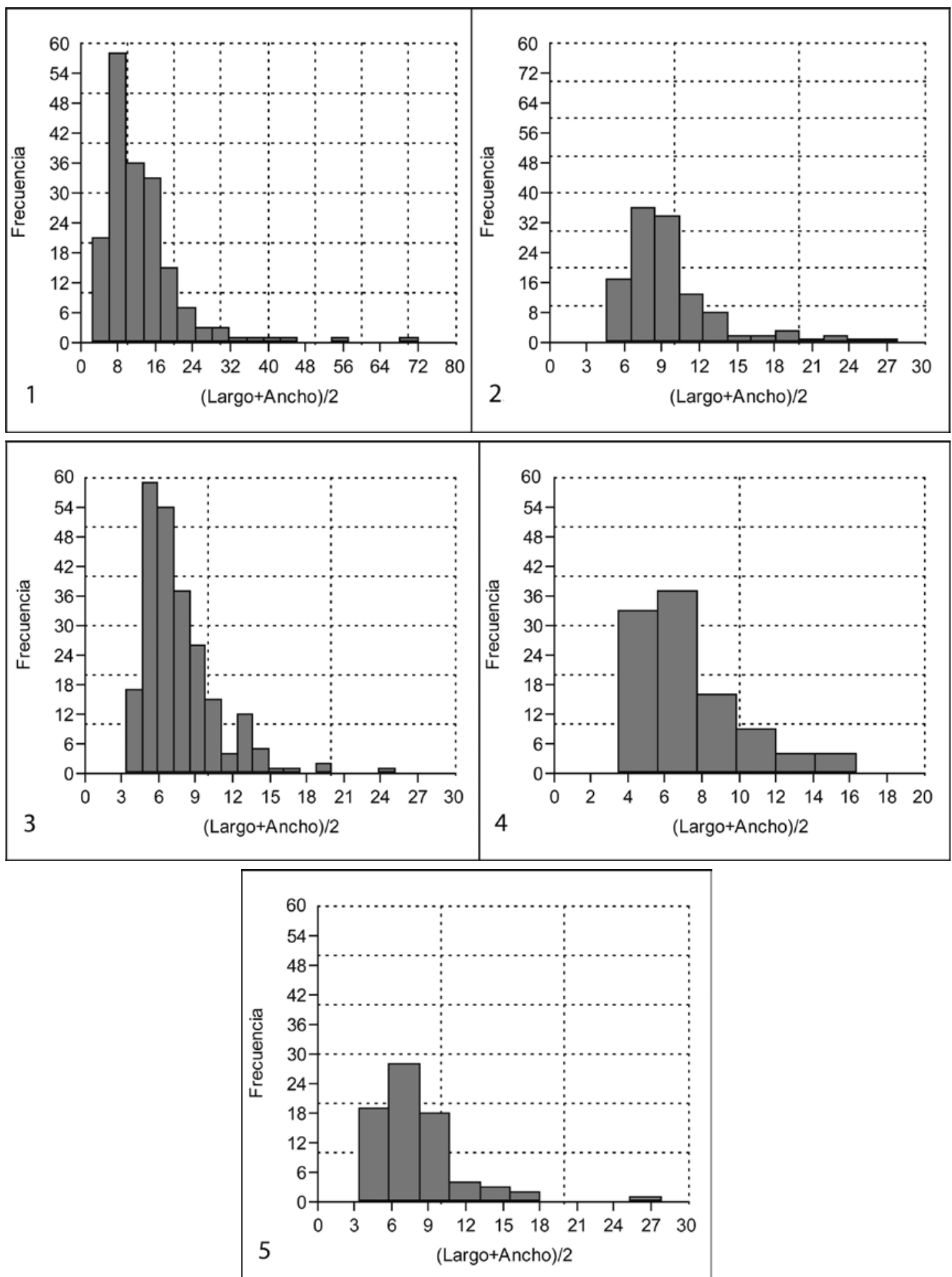

Figura 4. Tamaños de artefactos. 1. Cuarcita, 2. Andesita, 3. Obsidiana, 4. Rocas silíceas, 5. Cuarcita de grano fino. Size of artifacts. 1. Quartzite, 2. Andesite, 3. Obsidian, 4. Siliceous rocks, 5. Fine grain quartzite. 

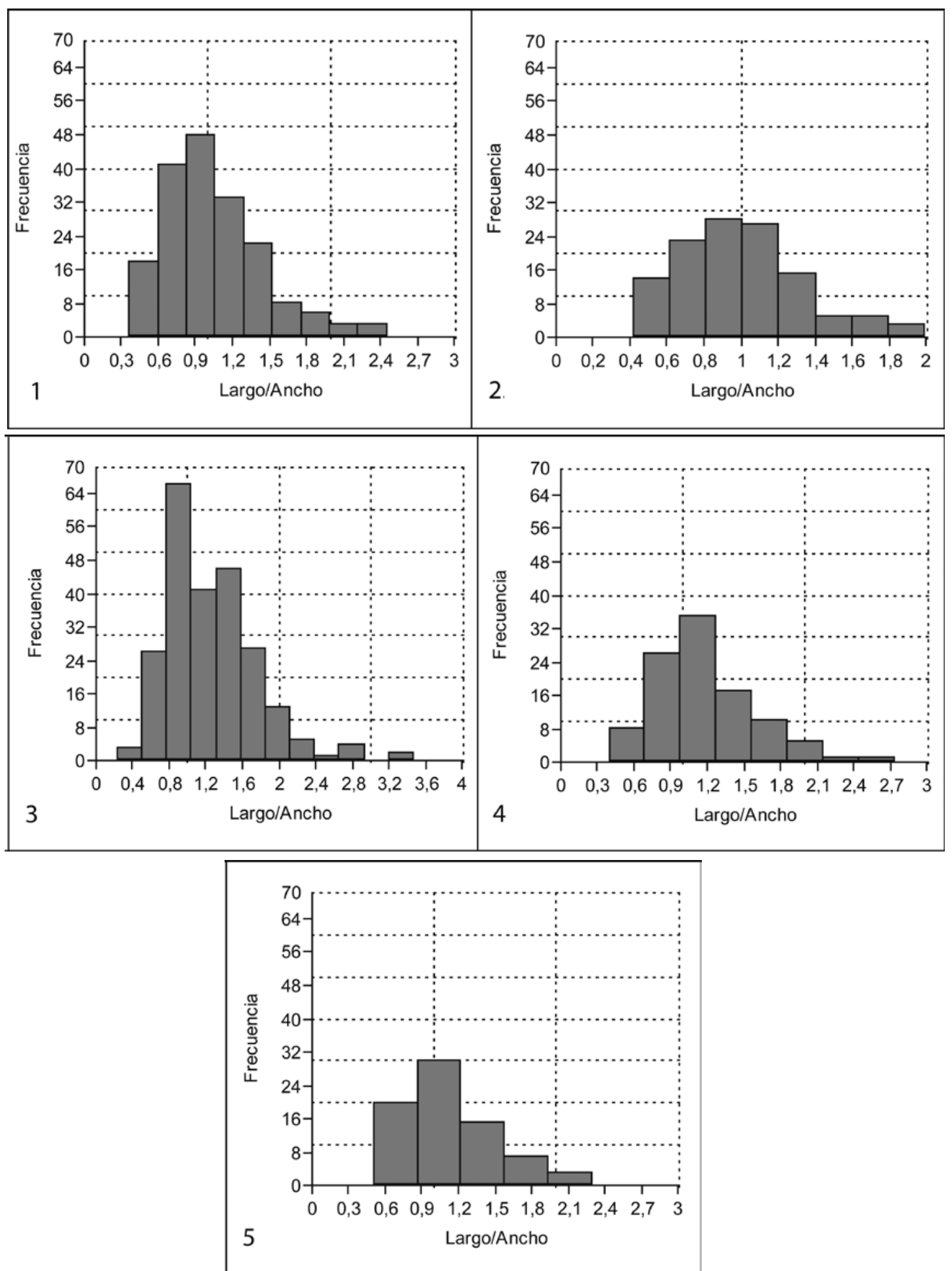

Figura 5. Módulos de artefactos. 1. Cuarcita, 2. Andesita, 3. Obsidiana, 4. Rocas silíceas, 5. Cuarcita de grano fino. Modules of artefacts. 1. Quartzite, 2. Andesite, 3. Obsidian, 4. Siliceous rocks, 5. Fine grain quartzite. 

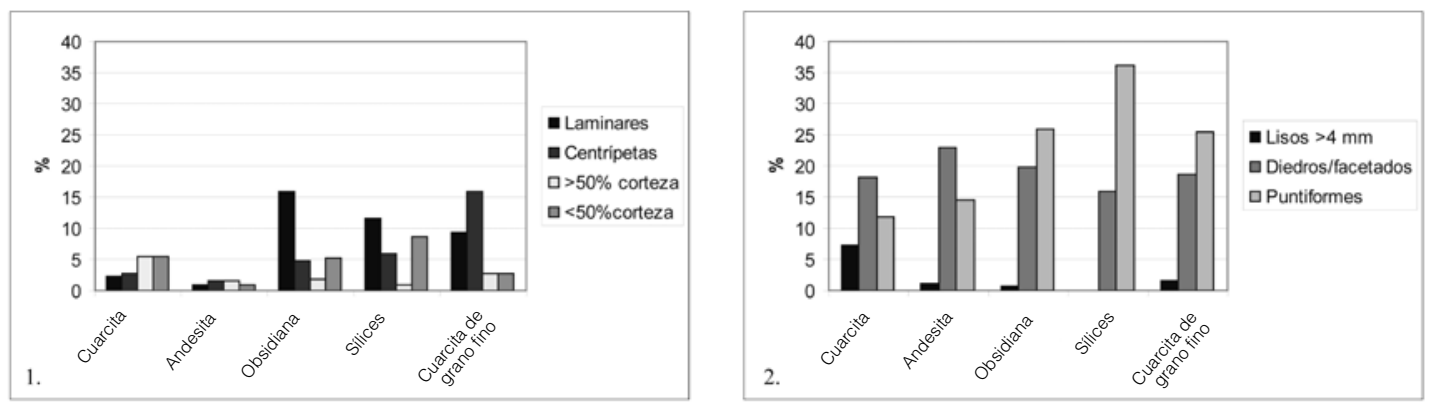

Figura 6. Porcentaje de los tipos de 1. Extracciones y 2. Talones por materia prima.

Proportion of types of 1. Debitage and 2. Platforms, by type of raw material.

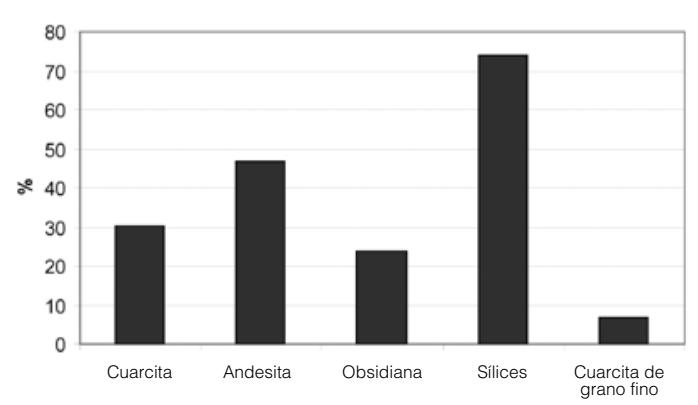

Figura 7. Porcentaje del peso de los instrumentos en relación al peso total por materia prima.

Weight percentage of tool types against total weight by raw material.

obsidiana, si bien se puede notar una importante frecuencia de módulos inferiores a 1, hay una proporción destacable de módulos alargados y laminares, con un $8 \%$ en este último caso, lo que no aparece para las demás materias primas (Figura 5.3). Los diagramas de frecuencias presentan casi la misma distribución para las rocas silíceas y las cuarcitas de grano fino (Figuras 5.4 y 5.5). En estos dos últimos casos hay una mayor frecuencia de módulos inferiores a $1, \mathrm{y}$ una cierta cantidad de artefactos presentan módulos alargados, con los laminares en muy baja frecuencia para estas dos materias primas.

Se puede notar que las proporciones más importantes de extracciones laminares aparecen en la obsidiana, luego en las rocas silíceas y finalmente en la cuarcita de grano fino (Figura 6.1). Al contrario, las extracciones centrípetas aparecen en mayores proporciones primero para la cuarcita de grano fino, luego para las rocas silíceas y finalmente para la obsidiana. Como se puede apreciar, las lascas con reserva de corteza presentan frecuencias muy bajas. Sumados los de menos y más de $50 \%$ de corteza el porcentaje más alto es el de la cuarcita, pero se puede notar la presencia significativa de lascas con menos de 50\% de corteza para las rocas silíceas y la cuarcita de grano fino.

Los talones diedros y facetados están bien representados para todas las materias primas y con proporciones parejas independientemente de cuál sea la roca (Figura 6.2). Presentan mayor énfasis para la cuarcita y la andesita mientras que los talones puntiformes tienen una proporción alta para los sílices, seguidos por las cuarcita de grano fino y por las obsidianas. Los talones lisos superiores a $4 \mathrm{~mm}$ están muy poco representados independientemente de cuál sea la materia prima, aunque la mayor proporción se observa en la cuarcita.

\section{Los Instrumentos}

Los instrumentos de andesita $(\mathrm{n}=4)$ representan el $47 \%$ del peso total (instrumentos + desechos) y los instrumentos de sílice $(n=3)$ el 74\% (Figura 7). Esto contrasta con las otras materias primas donde el peso de los instrumentos es mucho menor que lo representado por la abundancia del número de los desechos. Esto sugiere un descarte de instrumentos transportados al sitio pero confeccionados en otra parte. Al contrario, los muy bajos porcentajes de peso de instrumentos en obsidiana $(n=2,24 \%)$ y en cuarcita de grano fino $(n=1,7 \%)$ mostrarían que se confeccionaron instrumentos con estas materias primas en el sitio y que luego fueron llevados a otra parte.

Un percutor pulido de cuarcita con un machacado en la parte de contacto de percusión permite postular que se realizó percusión directa dura en el sitio.

En el sitio fueron halladas siete puntas tetragonales ( $47 \%$ del total de instrumentos), todas fragmentadas (Figura 8). Presentan aletas y pedúnculo no muy marcados, son de limbo triangular 

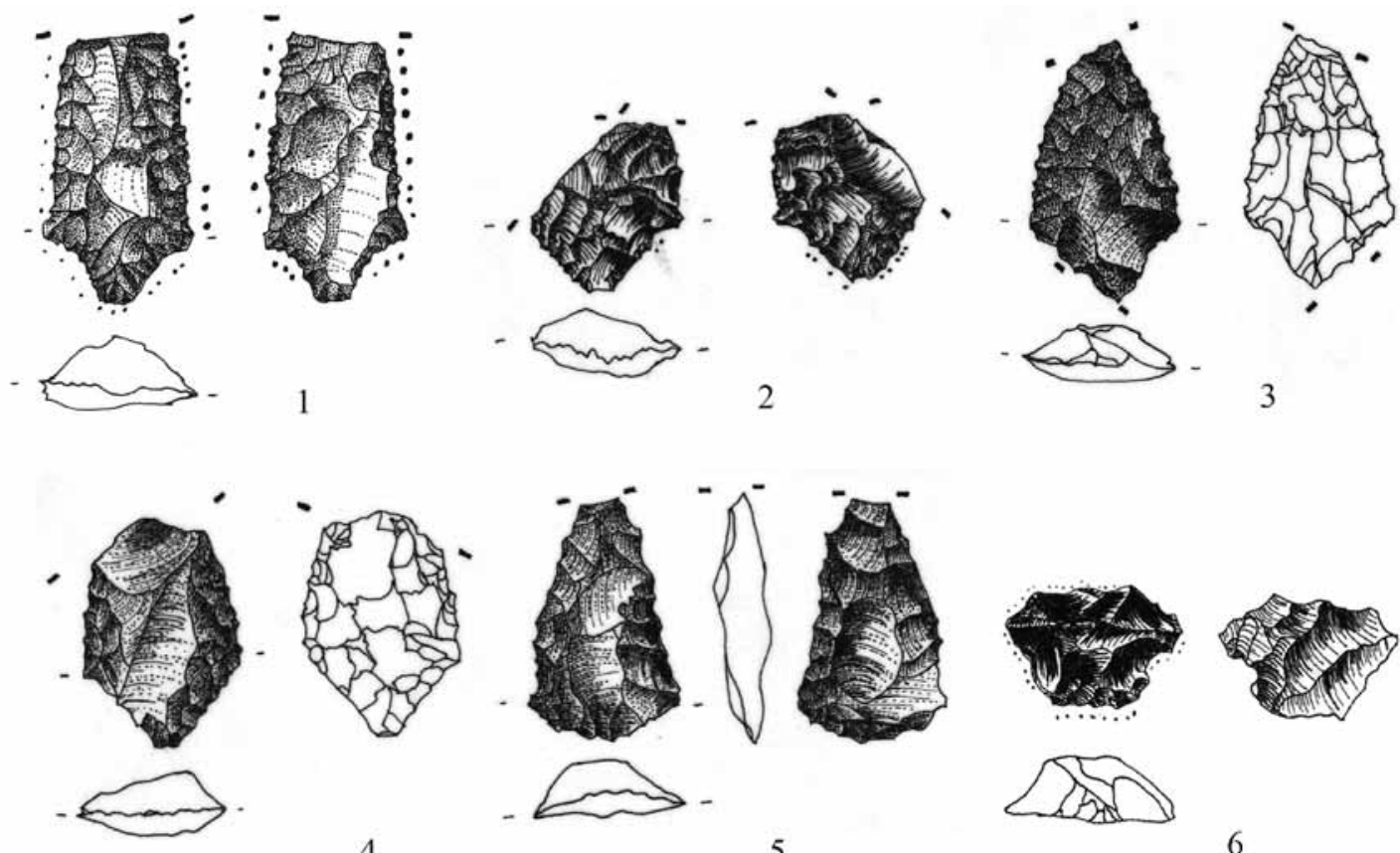

5
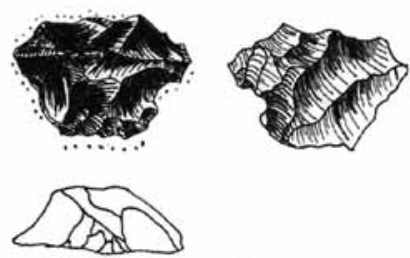

6

$5 \mathrm{~cm}$

Figura 8. Puntas tetragonales "San Martín" halladas en la capa 3 del alero Hornillos 2.

Tetragonal points "San Martín" from layer 3 of Hornillos 2 rockshelter.

y de sección plano-convexa. Todas son bifaciales, formatizadas con $\operatorname{dos}(\mathrm{n}=3)$ o tres secuencias $(\mathrm{n}=1)$ de adelgazamiento/retoque. Una de ellas parece haber sido un artefacto fallido (andesita) por no presentar rastros complementarios (Figura 8.5). Otra de estas puntas parece haber sido reciclada en un artefacto formatizado por extracciones paralelas en caras sucesivas (Figura 8.6). En cuanto a las materias primas, tres son de andesita, dos de obsidiana, una de calcedonia y una de cuarcita. Dos de ellas presentan ceniza adherida y rastros de rubefacción. Tienen diferentes modalidades de formatización, ya que están realizadas a partir de distintas secuencias. Las primeras extracciones realizadas en la forma-base apuntan a adelgazarla para obtener una preforma. Las preformas pueden ser unifaciales o bifaciales, las extracciones pueden invadir toda la cara o ser parcialmente extendidas y ser realizadas por extracciones esporádicas o por retoque escamoso escalonado. La última secuencia es en general un retoque marginal, paralelo muy regular, denticulado, bifacial o alterno (directo o indirecto) según el filo. Dos de ellas fueron intensamente recicladas, dada la cantidad de secuencias y la modificación morfológica del limbo en uno de los casos (Figuras 8.1 y 3).

Tres instrumentos fueron confeccionados sobre lascas grandes de cuarcita (más de $50 \mathrm{~mm}$ de tamaño) obtenidas por percusión dura. Uno presenta también dos filos, uno con rastros complementarios y el otro con retoque escamoso complementado por un microrretoque (Figura 9.1) cuya base presenta una cuidadosa preparación previa a su extracción (lasca predeterminada o forma-base). Otro presenta dos filos obtenidos por retoque inverso sobre una lasca secundaria ( $\sim 50 \%$ de corteza) (Figura 9.2). El último de estos instrumentos está fragmentado (mesial) y presenta un retoque bastante tosco, quebradizo, bifacial, acompañado de un microrretoque y rastros complementarios. Finalmente, debemos mencionar también un artefacto elíptico de andesita que fue adelgazado bifacialmente y completado con un microrretoque (Figura 9.3). Hay otros dos fragmentos de instrumentos indeterminados. 

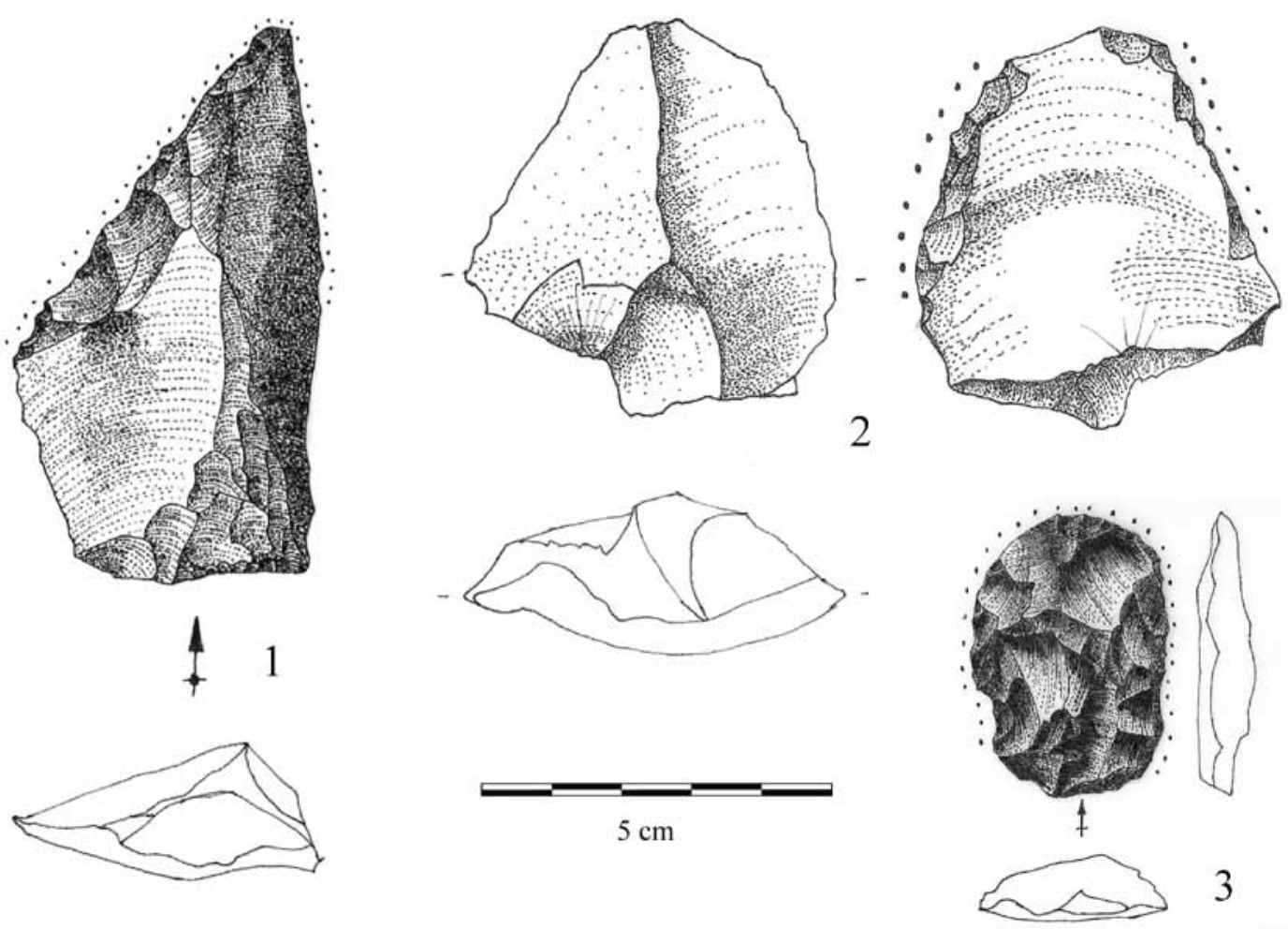

$5 \mathrm{~cm}$

Figura 9. Instrumentos varios (1 y 2 cuarcita, 3 andesita).

Selected tools (1 and 2 quartzite, 3 andesite).

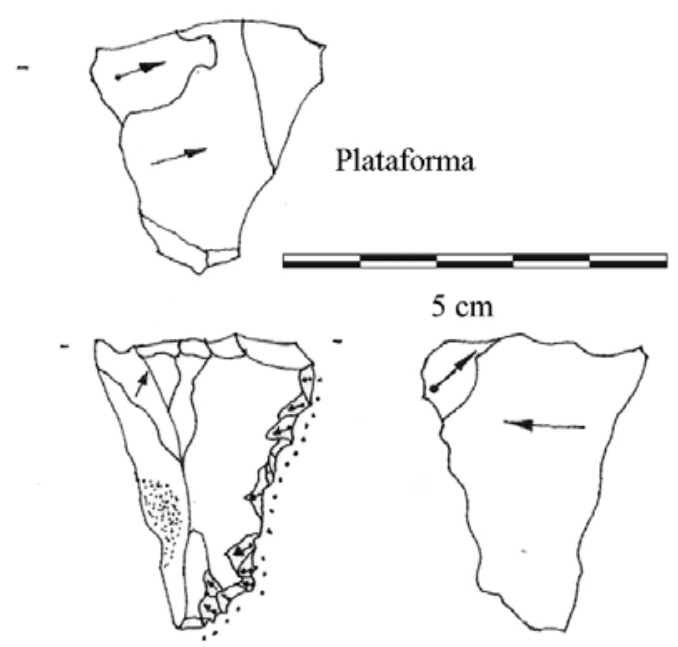

Figura 10. Núcleo de riolita retomado con retoque. Ryolite core rehabilitated with retouch.

En cuanto a los núcleos, se presentó uno de riolita fracturado, cuyo esquema de extracción resulta difícil de ordenar, y que fue retomado con un microrretoque denticulado (Figura 10). Otro pequeño núcleo de cuarcita presenta extracciones transversales.

\section{Discusión de los Resultados}

Las actividades de talla estuvieron predominantemente relacionadas a la formatización de artefactos, dada la alta presencia de tamaños comprendidos entre 5 y $12 \mathrm{~mm}$. Particularmente importantes parecen haber sido el retoque, el adelgazamiento de formas bases y la reactivación de instrumentos a fin de mantenimiento para casi todas las materias primas. Es interesante notar la alta proporción de desechos de obsidiana en relación al peso. Esto muestra que fue objeto de actividades intensas pero no dispendiosas debido, quizás, a las dificultades para su aprovisionamiento inmediato dada la lejanía de las fuentes de esta materia prima (Figura 1). Las rocas silíceas y la cuarcita de grano fino, a pesar de ser locales, fueron poco explotadas, seguramente por la baja disponibilidad. Pudieron haber sido trabajadas previamente en otros sitios 
a fin de transportarlos bajo forma de instrumentos, formas-bases y núcleos y que fueron abandonados después de su agotamiento.

Dada la abundancia de la cuarcita y de la disponibilidad de nódulos de varios tipos, lo primero que se puede destacar es la muy baja presencia de indicadores relacionados a la obtención de formas-bases y de formatización de núcleos. Fueron recuperadas algunas lascas grandes (superiores a $50 \mathrm{~mm}$ ) con o sin reserva de corteza y dos lascas de descortezamiento. El empleo de la técnica de percusión dura fue una actividad esporádica tanto para las primeras etapas de descortezamiento y formatización de núcleo -lascas predeterminando-, como para la obtención de formas-bases -lascas predeterminadas.

Según lo enunciado anteriormente respecto del peso y el tamaño de los desechos, se podría pensar el uso de la técnica de presión como el modo de talla principal para la obsidiana, dado que generó importante cantidad de desechos muy finos, lo que resultó en un peso muy bajo y, consecuentemente, en una optimización del uso de esta roca. Las importantes frecuencias de talones puntiformes, de módulos y de extracciones laminares concuerdan con el uso de la presión, observado, también, en las extracciones subparalelas o paralelas y poco profundas en los instrumentos de obsidiana presentes en el sitio.

Los distintos resultados muestran que la técnica por presión fue también utilizada para formatizar instrumentos en diversas rocas silíceas y en cuarcita de grano fino. Si su presencia no es tan clara en los desechos de cuarcita y de andesita, el retoque denticulado marginal paralelo de las puntas tetragonales muestra la intervención de esta técnica para esta secuencia, con un posible tratamiento térmico en el caso de la cuarcita (Pelegrin comunicación personal, 2010).

Se puede efectuar una distinción entre las técnicas aplicadas y las rocas según su distancia, su disponibilidad y las dimensiones de los nódulos. El adelgazamiento bifacial junto al retoque bilateral bifacial parece haber sido realizado en el sitio para todas las materias primas, como indica la frecuencia significativa de talones diedros y facetados.

Varias puntas tetragonales requirieron de esta modalidad antes de ser formatizadas por presión (Hoguin 2010). Además, los talones de los desechos muestran mayor énfasis de la primera etapa (adelgazamiento bifacial) sobre artefactos de cuarcita y de andesita (bloques grandes y disponibles), mientras que los desechos de las otras materias primas (bloques medianos, menos accesibles) muestran mayor énfasis en la última etapa de retoque final por presión. Varias lascas con extracciones centrípetas, particularmente en cuarcita de grano fino, en rocas silíceas y en obsidiana pudieron también ser resultantes del adelgazamiento de una preforma.

La baja frecuencia y el tamaño pequeño de los núcleos junto a la baja frecuencia de lascas predeterminando o predeterminadas nos informa sobre el rol del sitio dentro de una organización tecnológica general, donde se llevaron a cabo muy pocas actividades mayormente enfocadas a la formatización de instrumentos y a su reactivación. Por eso no podemos reconstruir esquemas operativos completos relacionados a la producción de las formas-bases. Podemos pensar que las puntas tetragonales así como otros instrumentos pudieron haber estado relacionados a un esquema operativo predeterminado como lo indican algunos indicios, tales como la preparación de cornisa en ciertos instrumentos (i.e. Figura 9:1) o aristas previas en la base de una punta tetragonal (Figura 2, primera punta a la izquierda), así como en la variabilidad de las modalidades de formatización relacionada con formas-bases predeterminadas (Hoguin 2010). De esta forma, la producción de estas puntas está relacionada con cadenas operativas complejas, de las cuales, en Hornillos 2, solo se realizaron secuencias de talla parciales. La variabilidad de los patrones de confección y la alta predeterminación en los esquemas de talla de las puntas San Martín reflejan la implementación de un saber-hacer y de habilidades adecuadas frente a distintos tipos de situaciones.

Cabe destacar que el diseño particular de las puntas tetragonales San Martín muestra claramente la búsqueda de una silueta con características morfométricas particulares, como un pedúnculo, dos aletas y dimensiones estandarizadas. Recordemos que es posible que los talladores de estas puntas adaptaran sus conocimientos y destrezas técnicas de forma flexible a fin de llegar al producto respetando las características mencionadas (Hoguin 2010). Esto nos podría llevar a pensar en un sistema de enmangamiento con requisitos aerodinámicos específicos. Estas puntas podrían ser la parte visible de este sistema técnico particular (sensu Inizan et al. 1995) que involucró no solamente las cadenas 
operativas descritas en este trabajo, sino también las del aprovisionamiento de la madera, la confección de astiles y el pegamento. De la misma forma, estos instrumentos requieren de gestos y técnicas específicas para su uso eficiente.

\section{Distribución Regional de las Puntas Tetragonales}

La dispersión de tal sistema técnico fue amplia, aunque no regional. Sin embargo, involucró numerosos sitios en Chile (Figura 1) y muchos menos en el Noroeste Argentino. En este último caso estas puntas aparecen solamente en la Puna Seca, y no están presentes en sitios próximos a Hornillos 2 como el Alero Cuevas ubicado en Pastos Grandes unos $140 \mathrm{~km}$ al sur (López 2008). Tampoco se mencionan estas puntas, ni fechados contemporáneos para sitios ubicados en el norte de la Quebrada de Humahuaca, como Inca Cueva 4 (Aschero 1984), Huachichocana III (Fernández Distel 1974) y Pintoscayoc 1 (Hernández Llosas 2000). Los fechados radiocarbónicos que marcan la distribución temporal de estas puntas son aún muy pocos. De acuerdo a los mismos, la expansión temporal de las puntas estaría comprendida entre ca 8.200 a.p. y 7.400 a.p.

Esta configuración espacial lleva a preguntarnos cuales son los orígenes de estas puntas y las razones de tal patrón espacial. Aclarar las cadenas operativas y analizar el resto de los instrumentos y desechos asociados recuperados en los otros sitios podría ser una línea de trabajo productiva para entender tal fenómeno. Una hipótesis posible puede elaborarse a partir del rango de aprovisionamiento de las obsidianas que en Hornillos 2 se restringe a partir del Holoceno Medio contando con un aprovisionamiento principal a partir de Zapaleri/ Laguna Blanca, abandonándose otras ubicadas al sur del sitio como Quirón, utilizada durante las ocupaciones del Holoceno Temprano (Yacobaccio 2010). Zapaleri/Laguna Blanca está ubicada próxima al punto limítrofe tripartito entre Chile, Argentina y Bolivia (Seelenfreund et al. 2010), que determinarían desde Hornillos 2 una dirección de movilidad principal siguiendo el eje norte-sur que coincide con la distribución que tienen estas puntas en el Norte de Chile (Núñez et al. 2005). Debemos recordar que estas puntas están asociadas a otros tipos morfológicos y, por lo tanto, parecen haber formado parte de complejos sistemas de armas y, quizás, como sugiere su distribución mayormente en lugares abiertos, fueron utilizadas para caza a cierta distancia.

\section{Conclusiones}

A pesar de la información limitada obtenida hasta ahora resulta valiosa en lo que atañe al conocimiento de las primeras ocupaciones del Holoceno Medio en la Puna Seca del Noroeste argentino. Al observar otras localidades como San Martín 4, Ascotán 4A (Núñez et al. 2005) o algunas terrazas de la Quebrada de Lapao como La Playa, las densidades de artefactos del alero Hornillos 2 parecen mínimas. Esto nos podría permitir pensar a Hornillos 2 como un lugar de ocupación corta por un grupo reducido y especializado (posiblemente en la caza, dado el porcentaje alto de descarte de las puntas), en el marco de una movilidad logística (Binford 1980), similar a como fue planteado para el caso de Quebrada Seca 3 en Antofagasta de la Sierra durante el Holoceno Medio (Pintar 1995).

Los resultados del análisis del material lítico aquí presentado indican la presencia de una porción de una organización tecnológica cuyas actividades principales se desarrollaron seguramente en otras localidades. Es conveniente recordar que las puntas están todas fragmentadas, algunas reactivadas o recicladas, maximizando así la vida útil de estos artefactos. Por otro lado, la mayor parte de las puntas halladas en el sitio son de materias primas no inmediatamente locales (obsidiana y andesita). Esto concordaría con el hecho de que las mismas podrían haber sido transportadas y abandonadas en el sitio, último lugar de una larga vida útil tras varios usos y reciclajes, situación concordante con la hipótesis planteada. Por otra parte, el alto porcentaje de peso en instrumentos de sílice y de andesita sostendría el argumento de un tipo de ocupación corta planificada, en la cual se espera que los instrumentos procedan de otro lugar y que parte de ellos hayan sido llevados ya confeccionados al sitio (Kent 1992). Además, la ocupación correspondiente a esta capa sería no solamente planificada para ser corta, sino que esta ocupación fue realmente corta (o una suma de eventos cortos), ya que no se generaron más desechos con estas materias primas. La baja densidad de artefactos, tanto desechos como instrumentos, así como la baja variedad de 
instrumentos, también sustentaría un sitio ocupado brevemente (redundante a lo largo de los años o no) con un esquema de actividades especializadas dentro de un sistema con una movilidad logística según el concepto de Binford (1980).

Sin embargo, nos permitieron considerar una organización en la cual se desarrollaron actividades específicas, quizás relacionadas con la búsqueda de presas y la preparación para la caza dada el carácter efímero de la ocupación (Catá 2004). Los instrumentos muestran largas historias de vida con reactivaciones intensas o reciclajes. Estos fueron objeto de mucha preparación por distintas secuencias caracterizadas por el empleo sucesivo de diferentes técnicas de talla y utilizadas en un amplio rango geográfico que incluyó diferentes localidades.

En este sentido este trabajo constituye un primer paso -que necesita ser ampliado en el futuro- en el estudio de los comportamientos técnicos y de las decisiones tecnológicas de los grupos humanos que poblaron la Puna Argentina a comienzos del Holoceno Medio.

Agradecimientos: Gracias a Marcelo Morales y a Ramiro Barbarena por sus lecturas atentas y sus críticas. Gracias a Donald Jackson por el envío de información y bibliografía. Agradecemos también a Jacques Pelegrin por sus observaciones sobre las técnicas, a Paz Catá por sus sugerencias sobre la fauna del sitio, a Patricia Solá por la información geológica. Este trabajo se hizo en el marco del proyecto UBACYT (F152) de la Universidad de Buenos Aires y del PIP3173CONICET. Finalmente, queremos agradecer a los evaluadores y al comité editorial de Chungara por sus comentarios pertinentes.

\section{Referencias Citadas}

Aschero, C.A. 1983. Ensayo para una clasificación morfológica de artefactos líticos. Apéndice A y B Cátedra de Ergología y Tecnología, Facultad de Filosofía y Letras, Universidad de Buenos Aires, Buenos Aires.

- _ _ 1984. El sitio ICC-4: un asentamiento precerámico en la Quebrada de Inca Cueva (Jujuy, Argentina). Estudios Atacameños 7:62-72.

- _ _ 1994. Reflexiones desde el Arcaico Tardío (6.000-3.000 A.P.). RUMITACANA 1:13-17.

Aschero, C.A. y J.G. Martínez 2001. Técnicas de caza en Antofagasta de la Sierra. Relaciones de la Sociedad Argentina de Antropología Tomo XXVI:215-241.

Binford, L.R. 1980. Willow smoke and Dogs'Tails: Huntergatherer settlement systems and archaeological site formation. American Antiquity 45:4-20.

Boëda, E. 1997. Technogenèse des systèmes de production lithique au Paléolithique inférieur et moyen en Europe occidentale et au Proche-Orient. Habilitation à diriger des recherches, Université Paris X, Nanterre.

Catá, M.P. 2004. Los primeros 4.000 años de ocupación humana en la Puna: cambios y tendencias en el consumo de la fauna. Actas del XV Congreso Nacional de Arqueología Argentina, pp. 155. Universidad Nacional de Río Cuarto, Río Cuarto.

Fernández Distel, A.A. 1974. Excavaciones arqueológicas en la Cueva de Huachichocana, Dep. de Tumbaya, Prov. de Jujuy, Argentina. Relaciones Tomo VIII:101-127.

Gamble, C. 1992. Exchange, foraging and local hominid networks. En Trade and Exchange in Prehistoric Europe, editado por C. Scarre y F. Healy, pp. 35-44. Oxbow Books, Oxford.

Hernández Llosas, M.I. 2000. Quebradas altas a través del tiempo: el caso de Pintoscayoc. Estudios Sociales del NOA 4(2):167-224.
Hoguin, R. 2010. Lithic technology, Chaînes Opératoires and cultural transmission. En Desde el Origen de las Especies: Perspectivas en Arqueología Evolutiva, editado por H.J. Muscio y M. Cardillo. BAR International Series, Oxford, en prensa.

Inizan, M.-L., M. Reduron, H. Roche y J. Tixier 1995. Technologie de la Pierre taillée. Préhistoire de la Pierre Taillée, Tome 4. CREP, Meudon.

Kent, S. 1992. Studying variability in the archaeological record: An ethnoarchaeological model for distinguishing mobility patterns. American Antiquity 57:635-660.

Le Paige, G. 1970. Industrias Líticas de San Pedro de Atacama. Técnicas y Tipologías de las Industrias Líticas. Editorial Orbe, Santiago.

López, G.E.J. 2008. Arqueología de Cazadores y Pastores en Tierras Altas: Ocupaciones humanas a lo largo del Holoceno en Pastos Grandes, Puna de Salta, Argentina. South American Series, BAR International Series, Oxford.

Marchand, G. 1999. La Néolithisation de l'Ouest de la France: Caractérisation des Industries Lithiques. BAR International Series, Oxford.

Morales, M. 2010. Arqueología Ambiental del Holoceno Temprano y Medio en la Puna Seca Argentina. Modelos Paleoambientales Multi-Escalas y sus Implicancias para la Arqueología de Cazadores-Recolectores. South American Archaeology Series, BAR International Series, Oxford, en prensa.

Núñez, L. y M. Grosjean 1994. Cambios ambientales pleistocenoholocénicos: ocupación humana y uso de recursos en la Puna de Atacama (Norte de Chile). Estudios Atacameños 11:11-24.

Núñez, L., M. Grosjean, B. Messerli y H. Schrelier 1997. Cambios ambientales holocénicos en la Puna de Atacama y sus implicancias paleoclimáticas. Estudios Atacameños 12:25-33. 
Núñez, L., M. Grosjean e I. Cartajena 2002. Human occupations and climate change in the puna de Atacama, Chile. Science 298:821-824.

- - - 2005. Ocupaciones Humanas y Paleoambientes en la Puna de Atacama. Universidad Católica del Norte, Taraxacum, San Pedro de Atacama.

Pelegrin, J. 1995. Technologie lithique: Le Châtelperronien de Roc-de-Combe (Lot) et de La Côte (Dordogne). Cahiers du Quaternaire $N^{\circ} 20$, CNRS Edition, Paris.

Pintar, E. 1995. Los conjuntos líticos de los cazadores holocénicos en la Puna Salada. Arqueología 5:9-24.

Schobinger, J. 1969. Prehistoria de Sudamérica. Editorial Labor, Barcelona.

Schobinger, J., J.R. Bárcena y J. Fernández 1974. Excavación arqueológica del sitio precerámico "Espinazo del Diablo", Mina Aguilar, Provincia de Jujuy. Relaciones Tomo VIII: 89-99.

Seelenfreund, A., M. Pino, M .Glascok, C. Sinclaire, P. Miranda, D. Pasten, S. Cancino, M.I. Dinator y J.R. Morales 2010. Morphological and geochemical analysis of the Laguna Blanca/
Zapaleri obsidian source in the Atacama Puna. Geoarchaeology: An International Journal 25:245-263.

Yacobaccio, H.D. 2010. Aprovisionamiento de obsidianas en cazadores recolectores surandinos. Ponencia presentada en XVII Congreso Nacional de Arqueología Argentina, Mendoza.

Yacobaccio, H.D., M.P. Catá, M.R. Morales, D. Joly y C. Azcune 2005. Ocupaciones humanas tempranas en la Puna de Atacama: el alero Hornillos 2, Susques (Jujuy). En Artefactos Líticos, Movilidad y Funcionalidad de Sitios en Sudamérica. Problemas y Perspectivas, editado por P. Escola y S. Hocsman. BAR Internacional Series, Oxford, en prensa.

Yacobaccio, H.D., P.S. Escola, F.X. Pereyra, M. Lazzari y M.D. Glascock 2004. Quest for ancient routes: Obsidian sourcing research in Northwestern Argentina. Journal of Archaeological Science 31:193-204.

Yacobaccio, H.D. y M. Morales 2005. Mid-Holocene environment and human occupation of the Puna (Susques, Argentina). Quaternary International 132:5-14. 
